\title{
Are Some Stellar Coronae Optically Thick?
}

\author{
C. J. SCHRIJVER, ${ }^{1}$ G. H. J. VAN DEN OORD, ${ }^{2}$ \\ R. MEWE, ${ }^{3}$ AND J. S. KAASTRA ${ }^{3}$ \\ ${ }^{1}$ Lockheed Palo Alto Research Laboratories, Palo Alto, CA 94304, USA \\ ${ }^{2}$ Sterrekundig Instituut, Utrecht, The Netherlands \\ ${ }^{3}$ Space Research Organization of The Netherlands, Utrecht, The Netherlands
}

We discuss the coronal spectra of a sample of cool stars observed with the spectrometers of the Extreme Ultraviolet Explorer (EUVE). The emission measure distributions show (a) a relatively weak component between $0.1 \mathrm{MK}$ and $1 \mathrm{MK}$, (b) a dominant component somewhere between $2 \mathrm{MK}$ and $10 \mathrm{MK}$, and (c) in all cases but one a component in the formal solution at temperatures exceeding $\approx 20 \mathrm{MK}$. Where this hot tail is not associated with a real hot component, it is a spurious result reflecting a lowered line-to-continuum ratio, which, for instance, may be the result of a low abundance of heavy elements or of resonant scattering in some of the strongest coronal lines. We suggest that in Procyon's corona photons in the strongest lines formed around a few million Kelvin undergo resonant scattering in a circumstellar medium, possibly a stellar wind. The flare spectrum of AU Mic suggests that resonant scattering may also occur in dense, hot flare plasmas. The electron densities of the 5-15 MK component are some three orders of magnitude higher than typical of the solar-like component around $2 \mathrm{MK}$; the volume filling factors of the hot components are therefore expected to be relatively small.

\section{Introduction}

The soft X-ray emission from stellar coronae has been measured by satellites such as EINSTEIN, EXOSAT, and, most recently, ROSAT. Most of these observations made use of either broad-band filters or of imaging proportional counters with a spectral resolution of order $\lambda / \Delta \lambda \approx 1$. Broad-band observations, however, constrain the temperature structure of stellar coronae only weakly. Observations with moderate to high spectral resolution are required to determine the "differential emission measure" (DEM), i.e., the weighting function $D(T) \equiv n_{\mathrm{e}} n_{\mathrm{H}} d V / d \log (T)$ which, together with the volume emissivity, measures the contribution of plasma of a given temperature to the emitted spectrum. Recently, EUVE has observed EUV spectra with a resolution of 0.5 to $2 \AA$, observing lines characteristic of temperatures in the range from $\sim 10^{5}$ to $\sim 10^{7} \mathrm{~K}$, and a few lines outside that interval. We analyzed the spectra of eight cool stars, presented in detail in papers by Mewe et al. (1995) and Schrijver et al. (1995). This paper discusses the main conclusions for all sources but AY Cet. The DEM inversion method is described in these proceedings by Mewe et al.

\section{The Temperature Structure of Coronae}

Fig. 1 shows the $D(T)$ curves for our sample (see also Table 1). In order to facilitate the comparison of these results for plasma around temperatures typical of the quiescent solar corona, the curves are normalized to a comparable level for the (peak) emission measure between 2 to $4 \mathrm{MK}$.

Procyon and $\alpha$ Cen both have a relatively strong tail extending to well below $1 \mathrm{MK}$. Their $D(T)$ are alike between $10^{5} \mathrm{~K}$ and $10^{7} \mathrm{~K}$. A scaled $D(T)$ for a solar active region is comparable to that of $\alpha$ Cen over a substantial temperature range.

Capella and $\xi$ UMa form another pair with comparable $D(T)$ : both have a component 

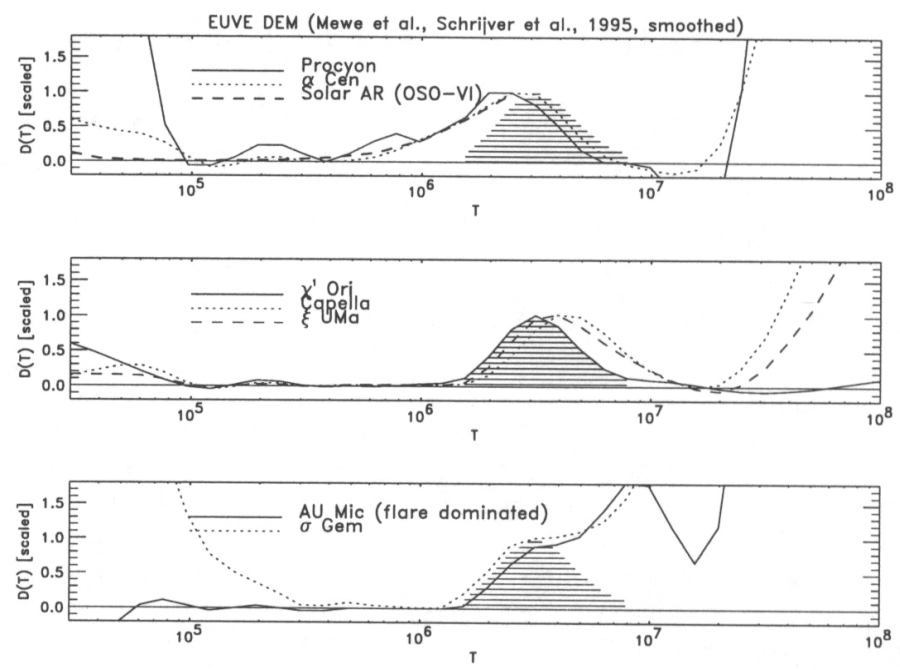

Figure 1. Comparison of $D(T)$ (as defined in Section 1; taken from Mewe et al. (1995), and Schrijver et al. (1995) -listed in Table 2 in the latter paper, which also gives the uncertainties). The curves are scaled to approximately unity around 2-4 MK, and are smoothed over two bins of $\log (T)=0.1$. The dashed region repeated in the three panels is the $D(T)$ of $\chi^{1}$ Ori between $1.5 \mathrm{MK}$ and $8 \mathrm{MK}$. The top panel also shows a differential emission measure curve for solar active region data observed with OSO-VI (from Dere (1982))

below $10^{7} \mathrm{~K}$ that extends to higher temperatures than those of $\chi^{1}$ Ori, Procyon, and $\alpha$ Cen, while they show little evidence of material with temperatures below about $1.5 \mathrm{MK}$.

The emission measure of $\sigma \mathrm{Gem}$ and AU Mic continues to increase above about $2 \mathrm{MK}$. Note that the spectrum of $\mathrm{AU}$ Mic is dominated by a major flare followed by a few smaller ones (see Brown \& Gagné et al. in these proceedings).

The $D(T)$ curves below $10^{5} \mathrm{~K}$ are dominated by the strong He II lines $256 \AA$ and 304 $\AA$. Their presence is indicative of upper chromospheric and transition-region emission, but as these lines are effectively thick, the $D(T)$ serves only as a formal solution.

All sources in our sample show a strong high-temperature tail, with the exception of the solar-like star $\chi^{1}$ Ori (GOV). The strong increase in $D(T)$ at very high temperatures, cut off in Fig. 1, corresponds to a total emission measure that exceeds the total emission measure of cooler plasma in the range from $\approx 20 \mathrm{MK}$ down to several hundred thousand Kelvin by factors of 2 up to 15 (see Table 1). This hot component is associated with a "continuum" in the SW range between $80 \AA$ and $140 \AA$ (that is observed in other cool stars too, see, for instance, the review by Brickhouse in these proceedings for another analysis of the Capella-spectrum, and the contributed paper by Walter on AR Lac). Even when assuming pure thermal equilibrium there are several plausible reasons for such a "continuum" to exist (also discussed by Schrijver et al. 1994 and 1995, Mewe et al. (1995), and in these proceedings by, e.g., Schmitt et al.): (1) an improper correction for the instrumental background; (2) a substantial number of closely spaced lines that are not (yet) included in the spectral code(s); (3) an optically thick continuum source; (4) a relatively low abundance for the elements producing most of the strong lines (predominantly $\mathrm{Fe}$ ); (5) an asymmetric plasma that is optically thick in the strongest lines; or (6) a real hot component. We rule out an improper background correction, because the shape of the apparent continuum does not conform with the gra- 
TABLE 1. Summary of results of emission-measure analyses by Mewe et al. (1995) and Schrijver et al. (1995)

\begin{tabular}{|c|c|c|c|c|c|c|}
\hline Source & $\begin{array}{l}T_{\max } \\
(\mathrm{MK})\end{array}$ & $\begin{array}{l}\mathrm{T} \text { 2nd } \\
\text { comp. } \\
(\mathrm{MK})\end{array}$ & $\begin{array}{l}\text { Hot } \\
\text { comp. } \\
\text { (rel.) }\end{array}$ & $\begin{array}{l}\text { Origin } \\
\text { of hot } \\
\text { comp. }\end{array}$ & $\begin{array}{c}\text { Electron } \\
\text { density } \\
\left(\mathrm{cm}^{-3}\right)\end{array}$ & $\begin{array}{c}\text { (Char. temp.) } \\
\text { Fe lines }\end{array}$ \\
\hline Procyon (F5IV-V) & 2 & $<1$ & 15 & thick? & $10^{9}-10^{10}$ & $\begin{array}{c}(1-2 \mathrm{MK}) \\
\mathrm{X}-\mathrm{XIV}\end{array}$ \\
\hline$\alpha \operatorname{Cen}(\underline{\mathrm{G} 2 \mathrm{~V}}+\underline{\mathrm{K} 2 \mathrm{~V}})$ & 3 & & 9 & thick? & $210^{8}-210^{9}$ & $\begin{array}{c}(1-2 M K) \\
X-X I V\end{array}$ \\
\hline $\mathrm{AU}$ Mic (MoVe) & 9 & 3 & 15 & $\begin{array}{l}\text { real\& } \\
\text { thick? }\end{array}$ & $2-510^{12}$ & $\begin{array}{l}(6-11 \mathrm{MK})^{*} \\
\text { XXI-XXII }\end{array}$ \\
\hline Capella (G5III+G0III) & 4 & & 3 & $\begin{array}{l}\text { real or } \\
\text { thick? }\end{array}$ & $10^{12}-210^{13}$ & $\begin{array}{l}(6-11 \mathrm{MK}) \\
\text { XIX-XXII }\end{array}$ \\
\hline$\sigma$ Gem $(\underline{\text { K1IIII}}+\ldots)$ & 15 & 5 & 2 & $\begin{array}{c}\text { real or } \\
\text { abund.? }\end{array}$ & $10^{12}$ & $\begin{array}{c}(10-20 M K) \\
\text { XXI-XXII }\end{array}$ \\
\hline$\xi \mathrm{UMa} \mathrm{B}(\underline{\mathrm{G} 0 \mathrm{~V}}+\ldots)$ & 4 & & 1.7 & $\begin{array}{l}\text { real or } \\
\text { abund.? }\end{array}$ & $510^{12}$ & $\begin{array}{c}(10-20 \mathrm{MK}) \\
\mathrm{XXI}-\mathrm{XXII}\end{array}$ \\
\hline
\end{tabular}

* Strongly weighted towards the initial phases of a strong flare.

NOTES: The component(s) of binaries expected to dominate the observed spectrum have been underlined. Note that the ratio of the emission measures in the tail above $28 \mathrm{MK}$ to that in the rest of the distribution above $0.9 \mathrm{MK}$ (above $0.1 \mathrm{MK}$ for Procyon), should be reduced by a factor of about four to six for a comparison in terms of counts if the continuum is interpreted as corresponding to the main component around about $3 \mathrm{MK}$ rather than to a component at over 10 MK (compare Schrijver et al. (1995)). The inferred densities are consistent with independent analyses of, e.g., Brickhouse, Gagné et al., Hanson et al., and Keenan et al. in these proceedings; see also further references in Mewe et al. (1995) and Schrijver et al. (1995).

dient in the instrumental background. Based on the EUVE spectra alone one cannot determine uniquely which (combination of) the remaining causes is responsible for the high-temperature tail in the $D(T)$ so that presently we rely on indirect arguments and tests of consistency. Additional information from instruments with other energy pass bands, such as $A S C A$, and EINSTEIN, however, can be used to exclude certain causes. Schmitt, Drake \& Stern (these proceedings) argue that for Procyon the $D(T)$-tail is incompatible with the ROSAT spectrum and can consequently not be real. A comparison with EXOSAT -TGS observations by Schrijver et al. (1995) resulted in the same conclusion. Schmitt et al. suggest that a number of lines missing from the spectral code are responsible for the "quasi-continuum." Although a number of weak lines are not included in the available spectral codes, it is doubtful that the missing lines are (1) sufficient in number and (2) have the appropriate strengths to mimic a flat continuum. The ensemble of missing lines should have the same effect in spectra of coronae as distinct as Procyon and AU Mic (flare dominated), but also have no effect in the spectrum of $\chi^{1}$ Ori. We therefore decided to investigate alternative options. We do not consider an optically thick continuum source a likely candidate. The last three options in the above list appear to us as the most viable: solar coronal abundances have been observed to differ from the photospheric abundances (see papers by Haisch and by J. J. Drake in these proceedings), while ever since the EINSTEIN mission a real hot component is known to be present in very active stars (see, e.g., Ayres, Brickhouse, Brown, Dupree et al., Gagné et al., and Walter in these proceedings).

Lowering the abundance of an element reduces the line-to-continuum ratio. Schrijver 
et al. (1995) tested the case of a reduced metal abundance for the stars in their sample. In each case the high-temperature tail is indeed reduced in strength by lowering the abundance (cf., Mewe et al. in these proceedings; see their Fig. 3), while the emission measure for the cooler plasma is increased as required in order to fit the emission lines now reduced in strength in the model. The quality of the fit does not, however, improve significantly. In the cases of $\sigma$ Gem and $\xi \mathrm{UMa}$, the quality of the fit with and without reduced iron abundances is about the same, with a reduced $\chi^{2}$-value below unity. Hence the possibility is left open in Table 1 that in some systems the tail may in fact be due to low iron abundances. The hot tail for AU Mic and Capella is likely to reflect a real hot component (see Schrijver et al. (1995) for details). For $\alpha$ Cen and Procyon, however, we suggest that resonant scattering in an asymmetric corona with line photon destruction upon impact on the stellar surface causes the spurious hot tail.

The usual assumption that stellar coronae are so tenuous that emitted photons escape without interaction with the stellar outer atmosphere or circumstellar plasma has been questioned for a number of strong lines based on solar observations (see below). A crude estimate of the optical depth can be made as follows: in the case of thermal Doppler broadening (which is likely to dominate in coronae) the optical depth at line centre is given by (e.g., Mariska 1992):

$$
\tau_{0}=1.210^{-17}\left(\frac{n_{\mathrm{i}}}{n_{\mathrm{el}}}\right) A_{\mathrm{Z}}\left(\frac{n_{\mathrm{H}}}{n_{\mathrm{e}}}\right) \lambda f \sqrt{\frac{M}{T}} n_{\mathrm{e}} \ell \equiv 10^{-19} C_{\mathrm{d}}\left(\frac{A_{\mathrm{z}}}{A_{\mathrm{z}, \odot}}\right)\left(\frac{n_{\mathrm{e}} \ell}{\sqrt{T_{6}}}\right)
$$

where $\left(n_{\mathrm{i}} / n_{\mathrm{el}}\right)$ is the ion fraction, $A_{\mathrm{Z}}=n_{\mathrm{el}} / n_{\mathrm{H}}$ the abundance, $n_{\mathrm{H}} / n_{\mathrm{e}} \approx 0.85$ the ratio of hydrogen to electron density (in $\mathrm{cm}^{-3}$ ), $\lambda$ the wavelength in $\AA, f$ the oscillator strength, $M$ the atomic weight, $T$ the temperature, and $\ell$ a characteristic dimension (in $\mathrm{cm}$ ). The product $n_{\mathrm{e}} \ell$, the column density, depends on the details of the coronal geometry. We tested two cases: a corona comprised of an ensemble of loops and a corona dominated by a spherically symmetric, hydrostatically settled envelope (see Table 2). The effective optical depth is strongly dependent on the geometry and temperature structure. In an elongated coronal loop photons suffer more scattering along the loop than perpendicular to it. In an actual stellar corona other loops overlay the originally emitting one, so that a photon can be scattered even if it escaped the loop in which it was originally emitted. The values for the loop-dominated atmosphere in Table 2 formally hold for radiation propagating along the loop, but in a more or less spherical volume of nested loops with the lowest most brightly emitting, the estimate approximates the net optical depth of coronal condensations over active regions.

Table 2 shows that some line-centre optical depths approach unity in loop-dominated geometries, particularly if sufficient loops overlie each other. But even for the values of the density and temperature for the base of the solar wind, optical depths can approach unity, while an increase in the base density or in the density scale height (as would be the case in a stellar wind) can raise these values. Hence, effects of resonant scattering should be expected in strong coronal emission lines.

\section{Resonant Scattering in the Solar Atmosphere}

Resonance scattering has been suggested many years ago for the solar corona. Based on observational data by Acton \& Catura (1976), Acton (1978) already investigated the problem of resonance scattering of X-ray emission lines in the solar corona. More recently, Rugge \& McKenzie (1985), for instance, discuss the effects of resonance scattering for the Fe XVII line ratios. Schmelz et al. (1992) use the resonance scattering of the $15.01 \AA$ Fe XVII line as a density diagnostic for solar active regions; they find that for a "typical" active region, over $50 \%$ of the photons for this resonance line could be scattered out of 
TABLE 2. Fe Resonance Lines

$\begin{array}{lrrlrrrr}\text { Ion } & \lambda(\AA) & T_{6} & f & b & C_{\text {d }} & \eta_{\text {oop }} & \tau_{\text {env }} \\ \text { Fe VIII } & \mathbf{1 6 7 . 4 9} & \mathbf{0 . 7} & 0.11 & 1.0 & 2.00 & 0.2 & 0.5 \\ \text { Fe IX } & 171.08 & 0.9 & 3.05 & 1.0 & 4.00 & 0.4 & 1.1 \\ \text { Fe X } & 174.53 & 1.1 & \mathbf{1 . 2 8} & 1.0 & 1.33 & 0.2 & \mathbf{0 . 4} \\ \text { Fe X } & 177.24 & 1.1 & 0.8 & 0.7 & 1.22 & 0.2 & 0.4 \\ \text { Fe XI } & 180.41 & 1.3 & 0.93 & 1.0 & 1.50 & 0.3 & 0.5 \\ \text { Fe XI } & 188.22 & 1.3 & 0.59 & 0.8 & 0.99 & 0.2 & 0.3 \\ \text { Fe XII } & 193.51 & 1.4 & 0.51 & 1.0 & 0.71 & 0.2 & 0.3 \\ \text { Fe XII } & 195.12 & 1.4 & 0.76 & 1.0 & 1.07 & 0.2 & 0.4 \\ \text { Fe XIII } & 197.43 & 1.6 & 0.73 & 0.3 & 1.49 & 0.4 & 0.6 \\ \text { Fe XIII } & 202.04 & 1.6 & 0.7 & 0.6 & 1.46 & 0.4 & 0.6 \\ \text { Fe XV } & 284.15 & 2.0 & 0.82 & 1.0 & 1.20 & 0.4 & 0.5 \\ \text { Fe XXI } & 128.73 & 8.9 & 0.09 & 0.9 & .091 & 0.3 & 0.1 \\ \text { Fe XXIII } & 132.85 & 12 . & 0.16 & 1.0 & .170 & 0.9 & 0.2 \\ \text { Fe XXIV } & 192.02 & \mathbf{1 5 .} & 0.05 & \mathbf{0 . 7} & .095 & 0.7 & 0.1 \\ \text { Fe XXIV } & \mathbf{2 5 5 . 1 0} & \mathbf{1 5 .} & 0.02 & \mathbf{0 . 3} & .047 & \mathbf{0 . 4} & 0.1\end{array}$

NoTES: Data for the strongest Fe resonance lines in the $\alpha$ Cen and AU Mic EUVE spectra (modified after Mewe et al. (1995), and Schrijver et al. (1995)), with line-centre optical depths $\pi_{\text {oop }}$ for a loop-dominated atmosphere and $\tau_{\text {env }}$ for a spherically symmetric gravitationally settled envelope of at least 0.3 . The value of $\eta_{\text {oop }}$ is computed using the scaling law for quasi-static coronal loops derived by Rosner et al. (1978). The value of $\tau_{\text {env }}$ is computed using a wind base density $n_{\mathrm{e}}=610^{8} \mathrm{~cm}^{-3}$, a temperature $T_{6}$ (in $\mathrm{MK}$ ) of the maximum line formation temperature, and a density scale height equal to the pressure scale height in a static, gravitationally stratified atmosphere, and the solar surface gravity. The envelope optical depth is proportional to the density scale height and to the base density, and inversely proportional to surface gravity. The loop optical depth depends on the topology and structure of the surrounding loops. Both quantities are therefore crude estimates. Also listed are the absorption oscillator strength, $f$, and the branching ratio, $b$. Solar photospheric abundances are used.

the line of sight, while optical depths range from 1.7 to 3 in their sample of four active regions. Waljeski et al. (1994) also study these lines as well as a few others. They show that the effects of resonance scattering for a pair of Fe XVII lines at $15.01 \AA$ and $15.26 \AA$ with substantially different oscillator strengths can be used as an abundance-independent density diagnostic. They also argue that O VIII at $18.97 \AA$ and Ne IX at $13.44 \AA$ also have optical depths exceeding unity for disk-center active regions. Note that the Fe XVII and $\mathrm{O}$ VIII lines strongly contribute to the spectra of stars as observed by the ROSAT PSPC, so that even in those data optical thickness effects need to be tested for, particularly in active stars where the emission is likely to be dominated by active-region sources.

Other data sets also support radiative-transfer effects in the solar corona. The simple examples in Fig. 2 show that as lines become stronger, the emission measure that is to be assigned to them to explain the observed total emission tends to decrease as the optical depth, compatible with resonant scattering as explained in the next section. We selected lines of $\mathrm{Fe}$ in a limited temperature range, thus eliminating abundance effects and excluding effects of strongly changing intrinsic emission measures with temperature. M. Laming pointed out that scattering in the quiet solar corona or base of the solar wind cannot be very strong, because for optical depths significantly larger than unity, off-limb structures would appear much more diffuse than actually observed (see, for instance, the images in Cheng (1980)). Hence, for the Sun, scattering inside the coronal condensations themselves seems to be the most important mechanism. 

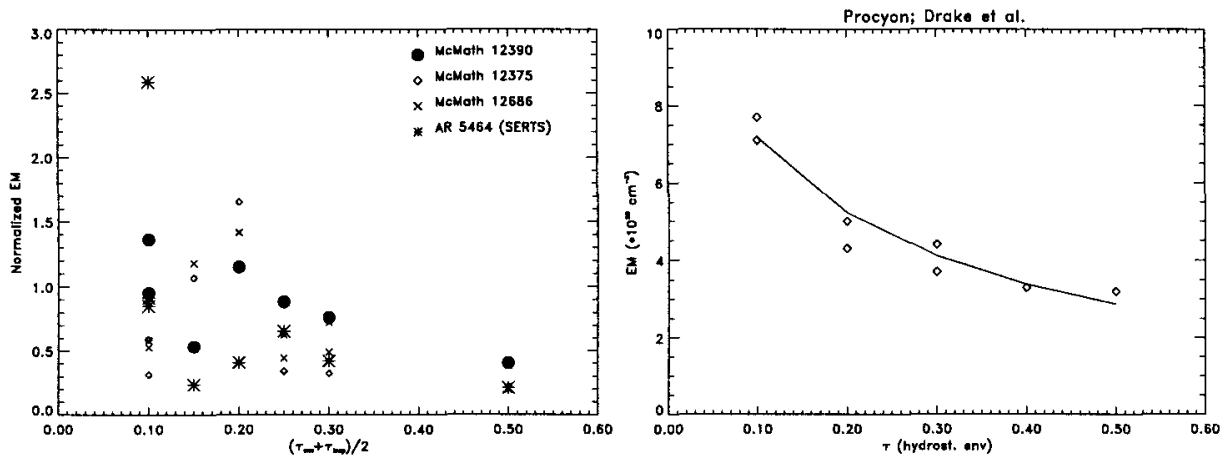

FIGURE 2. Left: Evidence for resonant scattering in the solar corona. Data for three active regions, observed at the solar limb with the NRL EUV slitless spectroheliograph, are taken from Dere (1982), and SERTS data for a fourth region from Thomas \& Neupert (1994). Plotted are normalized inferred emission measures, i.e., ratios of the observed line strengths for the strongest Fe XII through XV lines for which optical depths are listed by Mewe et al. (1995) to the emissivity from the Mewe et al. (1985) plasma code. The optical depths are taken from Table 2. Note that these are expected to differ from region to region and should be regarded as a relative measure only. The comparison of lines formed at different temperatures is made easier by a correction for the temperature dependence of the emission measure using the solar $D(T)$ plotted in Fig. 1. In order to allow the comparison of data for different regions, the EM values are scaled to unity for lines with optical depths (average of estimates for envelope and loop-dominated atmosphere) less then 0.26. McM 12375, McM 12686 and the SERTS region contained flaring or post-flare loops when observed, which probably accounts for part of the scatter. Right: Comparison of the estimated emission measure needed to explain observed line strengths for Procyon (from Drake et al. (1995)) compared to the expected optical depth in a hydrostatic coronal envelope with a temperature of roughly $2 \mathrm{MK}$ and a base density of $610^{8} \mathrm{~cm}^{-3}$ (as in the solar wind), as listed by Mewe et al. (1995). The lines from Fe XI up to Fe XVI range in wavelength from $192 \AA$ up to $359 \AA$, and are characteristic of temperatures from 1.3 MK up to $2.5 \mathrm{MK}$. The optical depth should be multiplied by a constant which depends on the actual base density and on the density scale height. (The curve shows the relationship $E M \propto 1 /(1+6 \tau)$, which Schrijver et al. (1995) argue to be indicative of scattering in a wind, although scattering in coronal condensations cannot be ruled out.)

These few references are not exhaustive, but they provide the reader with entry points into the solar literature, which does not appear to be substantially larger than the papers quoted here and the references therein; although the phenomenon of resonant scattering is known to solar coronal physicists, the complexity of the problem and the relative scarcity of extensive spectral data sets hampered developments in the field. Our discussion also shows, however, that existing data should be subjected to a careful analysis looking for evidence of a non-negligible optical thickness.

\section{Some Comments on Radiative Transport in Stellar Coronae}

Under coronal conditions, even repeated scattering rarely leads to photon destruction in the corona itself, because the probability of collisional de-excitation is very small. The scattered photons do, however, have a substantial chance of being destroyed upon impact on the stellar surface. If the scattering and emitting media were homogeneous and identical, any downward scattered photon would on average be compensated for by an upward scattering of an initially downward-emitted photon, so that no net effect would be observed. If, however, the emitting plasma lies, on average, below the scattering medium, an asymmetry is created in which more line photons are scattered down than 
up (see Schrijver et al. (1994)). As a result, the lines that weigh most strongly in the inversion process appear to be too weak compared to the continuum (not necessarily observable at the same wavelengths!) introducing a spurious hot component to $D(T)$. This model predicts that the strongest lines are affected most, as is indeed the case in Fig. 2. Note that the reduced scatter for Procyon as compared to the solar data in that Figure may be the result of (a) disk-averaging of the stellar emission, and (b) a reduced importance of individual flares on the mean stellar $D(T)$.

If resonant scattering is indeed important in stellar coronae, the implicitly assumed linearity of the $D(T)$-inversion problem breaks down, and detailed radiative transfer calculations will be required if all spectral information is to be used. All the usual problems complicating the recovery of the atmospheric structure from the observed emission that are encountered in the studies of stellar photospheres and chromospheres will have to be faced in coronal studies as well.

Frequency redistribution: The simple model discussed by Schrijver et al. (1994) is formulated for a single frequency, and therefore implicitly assumes that the resonant scattering is coherent. In reality significant frequency redistribution will occur. Thomas (1957), for instance, already argued that within the Doppler core of the line, the assumption of complete redistribution (CRD) over the thermal Doppler profile provides a very good approximation (see, for instance, discussions in Athay (1972), and Mihalas (1978)). With CRD and multiple scattering, line photons predominantly escape by "diffusion" in wavelength to a value where the optical depth becomes less than unity. One can define the probability $P_{\mathrm{e}}$ for photon escape as the fraction of the photons that have an optical depth less than unity as computed from the thermal absorption profile (e.g., Athay (1972)): $P_{\mathrm{e}}=\left(\tau_{0} \sqrt{\pi \ln \tau_{0}}\right)^{-1}$. For a line-center optical depth of $\tau_{0}=2$ to 5 , some $34 \%$ to $9 \%$, respectively, of the photons escape without suffering a single scattering simply because of the lower optical depth in the line wings. This limits the strength of the effects on the line-to-continuum ratio.

Anisotropy and geometry: The optical depth $\tau_{\nu}$ depends on the plasma which photons encounter in the outer atmosphere, and is therefore dependent on the detailed topology and the photon's path. In a spherical stellar atmosphere photons have a higher probability to escape in the radial direction resulting in an anisotropic distribution that is peaked in the direction of the normal (see Haisch \& Claflin (1985) for a discussion of these effects). If the distribution of emitting regions, possibly embedded in a scattering envelope, is not spherically symmetric, the number of photons escaping into the direction of the observer can be significantly affected.

\section{Electron Densities in Stellar Coronae}

The inferred electron densities listed in Table 1 and reported by others during the meeting (see caption to Table 1) suggest that the electron densities associated with the solar-like component at approximately $3 \mathrm{MK}$ are typically about three orders of magnitude lower than found for hotter components around 5-15 MK. Brickhouse (these proceedings) discusses evidence from the Capella-spectrum that this contrast is present within the same system. It is at present unknown whether the hot $5-15 \mathrm{MK}$ component is associated with hot loops or with flaring events, but regardless of this the high volume emissivity of the hot component implies that the volume filling factors of the hot components are much smaller than those associated with the $3 \mathrm{MK}$ component.

\section{Concluding Remarks}

The line-to-continuum ratio in the EUVE spectra of Procyon, AU Mic and $\alpha$ Cen and possibly Capella is suggestive of the occurrence of scattering of photons in the strongest 
spectral lines. In the case of Procyon this could be due to a stellar envelope or wind with a temperature of approximately 1-3 MK. For AU Mic and Capella we suggest that the compact hot structures are embedded in an equally hot but less dense environment, in the case of Capella possibly consisting of larger and therefore less dense quasi-static magnetic loops, while in the case of $\mathrm{AU}$ Mic the (predominantly) flaring plasma could be embedded in a hot, more tenuous plasma that itself may be either flare-related or part of a hot, quiescent coronal component. The shallow, dynamic convective envelope of Procyon and the flaring corona of AU Mic may result in coronae that are quite different from those of the Sun, so that a significant increase of the optical depth as compared to the solar corona may not come as a surprise. The fact that we also found evidence for line-photon scattering in the spectrum of $\alpha$ Cen is surprising, but solar observations suggest that upon careful examination the solar disk-integrated spectrum may also show effects of resonant scattering. Deeper $E U V E$ exposures and instruments like the $S U M E R$ and $C D S$ spectrographs on board $S O H O$, the ORFEUS instrument, the spectrometers planned for $A X A F$ and $X M M$, but even full-disk imaging as planned with $T R A C E$ can be used to test the role of resonant scattering in stellar coronae further. For such studies the study of lines and continuum together is crucial (see Mewe et al. in these proceedings). Whenever resonant scattering occurs, the $D(T)$ results for the corresponding stars should be interpreted as lower limits in the temperature range in which scattering is important.

We thank T. Ayres, J. Bruls, J. J. Drake, C. Jordan, M. Laming, D. Liedahl, $\dot{R}$. Rutten, J. Schmitt, R. Stern, and K. Strong for insightful comments.

\section{REFERENCES}

ACToN, L. 1978, ApJ, 225, 1069

Acton, L. \& Catura, R. 1976, Phil. Trans. Roy. Soc. London, A, 281, 383

ATHAY, R. 1972, Radiation Transport in Spectral Lines, Dordrecht: Reidel

Cheng, C. $1980, \mathrm{SPh}, 65,347$

DERE, K. 1982, SPh, 77, 77

Drake, J. J., Laming, J., \& Widing, K. 1995, ApJ, in press

Haisch, B. \& Claflin, E. 1985, SPh, 99, 101

MARISKA, J. T. 1992, The Solar Transition Region, Cambridge University Press

Mewe, R., KaAstra, J., Schrijver, C., van Den Oord, G., \& Alkemade, F. 1995, AA, 296,477

MiHalas, D. 1978, Stellar Atmospheres, San Francisco: Freeman

Rosner, R., Tucker, W., \& Vaiana, G. 1978, ApJ, 220, 643

Rugge, H. \& McKenZIE, D. 1985, ApJ, 279, 338

Schmelz, J., SABA, J., \& Strong, K. 1992, ApJL, 398, 115

Schrijver, C., Mewe, R., van den Oord, G., \& KaAstra, J. 1995, AA, in press

Schrijver, C., VAN Den OORD, G., \& Mewe, R. 1994, AA, 289, L23

SchriJver, C. J. 1993, AA, 269, 446

Thomas, R. 1957, ApJ, 125, 260

Thomas, R. J., Neupert, W. M. 1994, ApJS, 91, 461

Waljeski, K., Moses, D., Dere, K., Saba, J., Strong, K., WebB, D., \& Zarro, D. 1994, ApJ, 429, 909 